\title{
Sistema seletivo de corte de carga utilizando Fluxo de Potência Ótimo e Lógica Fuzzy
}

\author{
Fulvio Maschio*. Luciano C. Siebert** \\ Alexandre R. Aoki*** \\ * Companhia Paranaense de Energia, Curitiba, PR 80420-170 \\ Brasil (Tel: +55 41 3234-6821; e-mail:fulvio.maschio@gmail.com). \\ ** Delft University of Technology, Delft, 2628 CD Netherlands (e-mail: \\ siebert.luciano@gmail.com) \\ *** Universidade Federal do Paraná, Curitiba, PR 80060-000 \\ Brasil,(e-mail: araoki@gmail.com)
}

\begin{abstract}
The daily operation of the electrical system is subject to unforeseen events that can have serious consequences, such as the collapse of the whole system. For situations such as these, there are control processes involving load shedding in the distribution system, some of which are automatic and others manual. Among the manuals is the Manual Load Shed Plan where the distribution agents must shed the load within five minutes after a ONS request. This paper proposes a selective load shedding system using Optimum Power Flow to divide the load shed amount and Fuzzy Logic for the feeders prioritization. The use of continuity indicators, georeferenced variables and instantaneous power values of the feeders are the distinguishing factors of this proposed classification methodology, bringing a dynamic and spatial approach not found in the literature. The proposed model used real data from a system of 406 substations, 615 lines and 1962 feeders, reaching all the requested load sheds and avoiding unnecessary sheds in substations.
\end{abstract}

Resumo: A operação diária do sistema elétrico está sujeita a imprevistos que podem trazer graves consequências, como o colapso de todo o sistema. Para situações como essas, existem processos de controle envolvendo corte de carga no sistema de distribuição, sendo que alguns desses processos são automáticos e outros manuais. Entre os manuais está o Plano de Corte Manual de Carga (PCMC) onde os agentes de distribuição devem efetuar o corte de carga em até cinco minutos após a solicitação do Operador Nacional do Sistema Elétrico (ONS). Este artigo propõe um sistema seletivo de corte de carga utilizando Fluxo de Potência Ótimo (FPO) para distribuição do montante de corte de carga e Lógica Fuzzy para a priorização dos alimentadores. A utilização de indicadores de continuidade, variáveis georreferenciadas e valores de potência instantânea dos alimentadores são os diferenciais dessa metodologia de classificação, trazendo uma abordagem dinâmica e espacial não encontrada na literatura. O modelo proposto utilizou dados reais de um sistema de 406 subestações, 615 linhas e 1962 alimentadores, atingindo os resultados para todos os cortes de carga solicitados e eliminando cortes desnecessários em subestações.

Keywords: optimal power flow; fuzzy prioritizing; load shedding; manual load shed plan; distribution network.

Palavras-chaves: fluxo de potência ótimo; priorização fuzzy; corte de carga; PCMC; rede de distribuição.

\section{INTRODUÇÃO}

Historicamente, a demanda por energia elétrica tem um crescimento em longo prazo, $22,83 \%$ de aumento no consumo final de eletricidade entre 2008 e 2017 segundo a última edição do Balanço Energético Nacional (EPE, 2018). Com esse aumento, deve também aumentar a oferta de energia. Para manter o balanço entre demanda e oferta por energia deve haver um planejamento de longo prazo. O planejamento energético de curto prazo funciona de maneira diferente e está sujeito a imprevistos que interferem em sua dinâmica. Grandes eventos no Sistema Interligado Nacional (SIN) podem ter como consequências variação em sua frequência e tensão, e os centros de operação em tempo real devem agir de forma rápida e eficaz para evitar consequências mais graves, como o colapso do sistema e os seus riscos associados, apresentados por Bruch, Kuhn and Schmid (2011) e também evitar que tomem grandes proporções, como os eventos estudados por Veloza e Santamaria (2016). Para situações como essas, existem processos de controle envolvendo corte de carga no sistema de distribuição de média tensão, prevenindo o colapso do SIN, sendo que alguns desses processos são automáticos e outros manuais.

A ANEEL (2015) define corte de carga como sendo uma interrupção de suprimento de energia elétrica através do 
desligamento automático ou manual, de linhas de transmissão ou de circuitos de distribuição. O Operador Nacional do Sistema Elétrico (ONS) define o gerenciamento da carga, através do Submódulo 10.10, como atividades em tempo real voltadas a cobrir qualquer deficiência de geração, transmissão ou transformação em que a carga a ser atendida supere a capacidade de suprimento/atendimento da área afetada resultando, portanto, em remanejamentos ou cortes de carga previamente estabelecidos para garantia da integridade do sistema (ONS, 2016). O gerenciamento da carga também contribui para a segurança do sistema e para manter a qualidade do fornecimento de energia. Entre os automáticos está o Esquema Regional de Alívio de Carga (ERAC), e entre os manuais estão o Plano de Corte Manual de Carga (PCMC) e os planos de corte regionais (ONS, 2016).

A solicitação para gerenciamento de carga pelo PCMC poderá ser feita mesmo que ocorra atuação do ERAC, caso a subfrequência se mantenha de forma sustentada abaixo do valor nominal após atuação desse esquema.

O objetivo deste trabalho é apresentar os resultados obtidos do sistema seletivo de corte de carga pela metodologia proposta utilizando o Fluxo de Potência Ótimo (FPO) para distribuição do montante de corte de carga e Lógica Fuzzy para a priorização dos alimentadores, trazendo objetividade frente a uma classificação puramente subjetiva baseada na experiência prática do analista responsável por esta atividade, aprimorando os métodos utilizados atualmente.

O restante desse artigo está organizado da seguinte maneira: na seção 2 são apresentadas as práticas atualmente adotadas por algumas distribuidoras para o PCMC, na seção 3 o método proposto com a utilização de FPO + FUZZY é explicado, com seus resultados apresentados e discutidos na seção 4. Na seção 5 as conclusões são sintetizadas.

\section{PRÁTICAS ADOTADAS PARA O PCMC}

O PCMC deve agrupar os cortes em patamares de até 5\%, até o montante de $35 \%$ de sua carga total em cada ponto de conexão e/ou suprimento. Esta modulação em patamares de até $5 \%$ da carga permite tanto o atendimento a uma situação de corte de carga sistêmico, como também o atendimento a uma contingência localizada no sistema de transmissão ou transformação. Deve ser aplicável a todos os patamares de carga, e também explicitar as cargas selecionadas para o corte, tendo como referência os pontos de conexão e/ou suprimento destas nas instalações.

Os procedimentos operacionais de gerenciamento de carga para o controle da frequência estão descritos na instrução IOGC.BR.01 (ONS 2018b). Quando este recurso é utilizado, os agentes de distribuição devem efetivar o corte de carga em até 5 minutos após a solicitação do ONS. No anexo 1 da instrução de operação estão relacionados o montante de carga a ser cortada por agente em função da frequência do SIN.

O restabelecimento das cargas cortadas deverá aguardar autorização do ONS, que o fará de forma gradativa após a frequência atingir $60 \mathrm{~Hz}$ e permanecer estabilizada por pelo menos um minuto.
Atualmente a Copel é dividida em cinco regiões e todas as subestações são automatizadas através do Sistema de Automação de Subestações (SASE). Através dos dados coletados pelo SASE e informações dos consumidores agrupados por classe de consumo, até 2018 era aplicada uma equação cujo resultado é uma tabela que relaciona todos os alimentadores e seus respectivos graus de prioridade de corte.

A tabela de priorização de corte de carga divide os alimentadores em blocos de carga de $25 \mathrm{MW}$, para cada uma das cinco regiões do Paraná, nos patamares de carga leve, média e pesada. Quando solicitado, o montante de corte de carga é distribuído proporcionalmente pela carga de cada região (divisão proporcional pela região geoelétrica).

A Copel está desenvolvendo uma nova metodologia através do Programa de Pesquisa e Desenvolvimento da ANEEL projeto PD 2866-0404/2014 - CORTE SELETIVO DE CARGA EM CONSUMIDORES E ALIMENTADORES DE DISTRIBUIÇÃO.

\subsection{Práticas adotadas pela EDP Bandeirante}

Para atender o PCMC de maneira eficaz, a EDP Bandeirante desenvolveu um sistema que faz o gerenciamento do corte de carga através do desligamento dos alimentadores e a emissão automática de relatórios com as cargas cortadas. O Esquema de Corte para Alívio de Carga (ECAM) foi implementado no sistema de supervisão e controle do Centro de Operações. (Silva, Marques and Jardim, 2016)

A priorização dos alimentadores é feita através do número de clientes prioritários cadastrados no alimentador, e quando não houver nenhum, é adotado o critério de impacto social, uma relação entre carga típica pelo número de clientes afetados.

\subsection{Práticas adotadas pela Cemig Distribuição}

Atualmente a área de concessão da Cemig é dividida em sete regiões. Seu Sistema de Aquisição de Grandezas Analógicas (SAGA) trabalha em conjunto com o atual Sistema de Supervisão e Controle (SSC) do Centro de Operação de Distribuição (COD). O módulo automático do PCMC possui uma tabela de cargas separadas em regiões geográficas do estado de Minas Gerais, com uma lista de prioridade para o corte das cargas. Em sua tela principal o Técnico de Operação do Sistema preenche o total de carga a ser cortado e pode alterar os percentuais de carga por região, que já estão preenchidos com valores padrão. O resultado é uma sequência de manobras para desligar que ao ser executada efetuará o corte programado e também uma sequência de manobras para religar que ao ser executada religará as cargas na sequência inversa ao do desligamento. (Vieira et al., 2016)

\section{MÉTODO PROPOSTO FPO + FUZZY}

Este artigo propõe um sistema seletivo de corte de carga utilizando FPO para distribuição do montante de corte de carga e Lógica Fuzzy para a priorização dos alimentadores.

\subsection{Distribuição do montante de corte de carga através do FPO}

\subsection{Práticas adotadas pela Copel Distribuição}


Para a distribuição do montante de corte de carga foi utilizado o método apresentado por (Mikilita, 2005) através de um algoritmo primal-dual de pontos interiores, utilizando como dados de entrada os dados disponíveis do ANAREDE do sistema de transmissão e distribuição de alta tensão do estado do Paraná, totalizando um sistema de 406 barras e 615 linhas.

Para as barras das subestações foram utilizados os seguintes dados: identificação, tipo de barra, tensão, ângulo de fase, potência de geração ativa e reativa, limite mínimo e máximo de geração reativa, potência de carga ativa e reativa, capacitor reator, e área a qual a barra pertence. Para os circuitos foram utilizados os seguintes dados: identificação das barras nas extremidades, identificação do circuito, resistência, reatância, susceptância, tap atual, taps mínimo e máximo, barra controlada (quando transformador) e capacidade de carregamento em condições normais e em emergência.

Foram utilizados os valores de $\mathrm{V}_{\mathrm{i}} \min =0,85$ p.u. e $\mathrm{V}_{\mathrm{i}} \max =$ 1,05 p.u. como limites de tensão nas barras para minimizar os impactos do corte de carga no perfil de tensão do sistema e utilizado o limite de 0,6 para o máximo corte por barra para evitar o corte total de uma subestação, que em algumas regiões pode representar uma ou mais cidades.

\subsection{Priorização dos alimentadores através da Lógica Fuzzy}

Foram utilizados os seguintes dados de entrada de 1962 alimentadores do sistema de distribuição de média tensão do estado do Paraná para o desenvolvimento da lógica fuzzy: consumidores por classe de consumo, potência ativa instantânea e máxima dos últimos 12 meses, Consumidor x Hora Interrompido (CHI) dos últimos 3 meses, Consumidor Interrompido (CI) dos últimos 3 meses e dados georreferenciados para o cálculo da distância do alimentador até o centro da região. Para alguns conjuntos fuzzy houve a necessidade de utilizar seus dados normalizados, para garantir uma escala fixa entre 0 e 1 , utilizando então a equação (1)

$\mathrm{DADOent}_{A L I M}=\frac{\operatorname{VALOR}_{A L I M}}{\operatorname{VALOR}_{M A X}}$

Onde:

DADOent $_{\mathrm{ALIM}}$ é o dado de entrada utilizado no conjunto fuzzy para o alimentador, com valor entre 0 e 1 .

VALOR $_{\text {ALIM }}$ é o valor absoluto da variável do alimentador.

VALOR $_{\mathrm{MAX}}$ é o valor absoluto máximo da variável entre todos os alimentadores.

Utilizando como referência os trabalhos de Reddy et al. (2017), Calderaro et al. (2011) e Ganeshprasad et al. (2013), os conjuntos fuzzy foram então organizados da seguinte maneira:

a) Potência instantânea do alimentador - os conjuntos foram definidos de acordo com seu potencial de contribuição ao corte de carga, e suas transições foram dimensionadas de modo a traduzir aproximadamente a avaliação do analista responsável pela classificação dos alimentadores, sendo definidos da seguinte maneira: Baixa $=\left[\begin{array}{lll}-10 & -10 & 1\end{array}\right]$, Média $=\left[\begin{array}{llll}1 & 4 & 6 & 12\end{array}\right]$ e Alta $=\left[\begin{array}{llll}6 & 12 & 20 & 20\end{array}\right]$, como mostra a Figura 1.

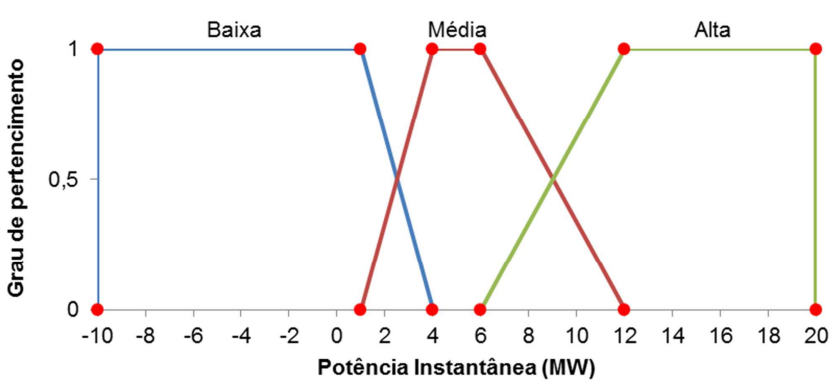

Fig. 1 Funções de pertinência para potência instantânea

b) Potência máxima do alimentador - os conjuntos e suas transições foram analisados de maneira análoga à potência instantânea do alimentador, resultando em: Baixa $=\left[\begin{array}{ll}-10 & -10\end{array}\right.$ 14 , Média $=\left[\begin{array}{llll}1 & 4 & 6 & 12\end{array}\right]$ e Alta $=\left[\begin{array}{llll}6 & 12 & 20 & 20\end{array}\right]$

c) Industriais - além do número de consumidores industriais, devido às características de consumo, também são incluídas as UCs cadastradas como serviços públicos e poderes públicos ligados ao alimentador. As transições dos conjuntos fuzzy "Poucos" e "Muitos" foram analisadas de maneira que aproximadamente $10 \%$ dos alimentadores ficassem acima da interseção dos conjuntos. Aplica-se a equação (1) para a normalização deste dado de entrada. Os conjuntos ficaram definidos como: Poucos $=\left[\begin{array}{lll}0 & 0 & 0,45\end{array}\right]$ e Muitos $=\left[\begin{array}{llll}0 & 0,45 & 1\end{array}\right]$.

d) Comerciais - além do número de consumidores comerciais, devido às características de consumo, também são incluídas as UCs cadastradas como próprios da concessionária ligados ao alimentador. A transição dos conjuntos fuzzy "Poucos" e "Muitos" foram analisados de maneira que aproximadamente $20 \%$ dos alimentadores ficassem acima da interseção dos conjuntos. Aplica-se a equação (1) para a normalização deste dado de entrada. Os conjuntos ficaram definidos como: Poucos $=\left[\begin{array}{lll}0 & 0 & 0,35\end{array}\right]$ e Muitos $=\left[\begin{array}{llll}0 & 0,35 & 1 & 1\end{array}\right]$.

e) Residenciais - além do número de consumidores residenciais, devido às características de consumo, também são incluídas as UCs cadastradas como rurais, iluminação pública ou sem cadastro, ligados ao alimentador. As transições dos conjuntos fuzzy "Poucos", "Médio" e "Muitos" foram analisados de maneira que aproximadamente $20 \%$ dos alimentadores ficassem acima da interseção dos conjuntos "Médio" e "Muitos" e aproximadamente 30\% dos alimentadores ficassem abaixo da interseção dos conjuntos "Poucos" e "Médio". Aplica-se a equação (1) para a normalização deste dado de entrada. Os conjuntos ficaram definidos como: Poucos $=\left[\begin{array}{lll}0 & 0 & 0,16\end{array}\right]$, Médio $=\left[\begin{array}{lll}0 & 0,2 & 0,4\end{array}\right] \mathrm{e}$ Muitos $=\left[\begin{array}{llll}0,24 & 0,4 & 1 & 1\end{array}\right]$.

f) CHI - A transição dos conjuntos fuzzy "Baixo" e "Alto" foram analisados de maneira que aproximadamente $20 \%$ dos alimentadores ficassem acima da interseção dos conjuntos. Aplica-se a equação (1) para a normalização deste dado de entrada. Os conjuntos ficaram definidos como: Baixo $=\left[\begin{array}{ll}0 & 0\end{array}\right.$ $0,030,11]$ e Alto $=[0,030,1111]$. 
g) CI - A transição dos conjuntos fuzzy "Baixo" e "Alto" foram analisados de maneira que aproximadamente $20 \%$ dos alimentadores ficassem acima da interseção dos conjuntos. Aplica-se a equação (1) para a normalização deste dado de entrada. Os conjuntos ficaram definidos como: Baixo $=\left[\begin{array}{ll}0 & 0\end{array}\right.$ $0,060,14]$ e Alto $=[0,060,1411]$.

h) Distância do alimentador - A transição dos conjuntos fuzzy "Perto" e "Longe" foram analisados de maneira que apenas alimentadores que estivessem em um raio de $10 \mathrm{~km}$ fossem considerados perto, ou seja, próximo a uma região de maior movimento. Os conjuntos ficaram definidos como: Perto $=[0$ 02 18] e Longe $=$ [2 18350 350] .

Uma vez definidos os conjuntos, as regras de inferência fuzzy foram determinadas seguindo as seguintes premissas:

a) Utilização de condição de barreira onde o corte é menos prioritário em alimentadores com $\mathrm{CHI}$ ou $\mathrm{CI}$ em conjunto fuzzy "Alto", para evitar que a satisfação percebida do consumidor seja afetada.

b) Utilização de condição de barreira onde o corte é menos prioritário em alimentadores de regiões centrais, isto é, com distância em conjunto fuzzy "Perto", para minimizar as consequências do corte de carga.

c) Alimentadores com Potência Instantânea em conjunto fuzzy "Alta" são mais prioritários para o corte de que os alimentadores em conjunto fuzzy "Média" e "Baixa", para atingir o corte de carga com menor número de alimentadores. De maneira análoga, alimentadores em conjunto fuzzy "Média" são mais prioritários para o corte de que os alimentadores em conjunto fuzzy "Baixa".

d) Alimentadores com Potência Máxima em conjunto fuzzy "Alta" são mais prioritários para o corte de que os alimentadores em conjunto fuzzy "Média" e "Baixa", porque possuem potencial de corte de carga maior. De maneira análoga, alimentadores em conjunto fuzzy "Média" são mais prioritários para o corte de que os alimentadores em conjunto fuzzy "Baixa".

e) Alimentadores com número de consumidores em conjunto fuzzy "Pouco" são mais prioritários para o corte de que os alimentadores em conjunto fuzzy "Médio" ou "Muito", para afetar o menor número de consumidores. De maneira análoga, alimentadores em conjunto fuzzy "Médio" são mais prioritários para o corte de que os alimentadores em conjunto fuzzy "Muito".

f) A variável de entrada Industriais é menos prioritária que as variáveis Comerciais e Residenciais.

g) A variável de entrada Comerciais é menos prioritária que a variável e Residenciais.

E por fim foram determinados os conjuntos de saída fuzzy (Fig. 2), utilizando o método centroide para posterior defuzificação e obtenção do valor de SF.

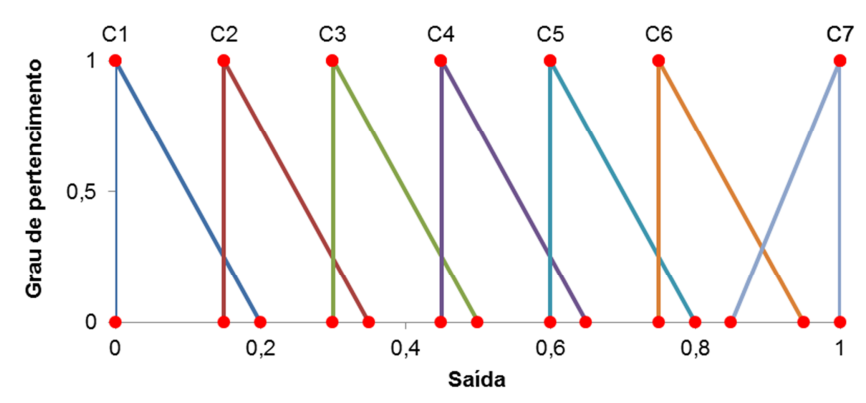

Fig. 2 Funções de pertinência para variável de saída

Para obtenção do indicador de prioridade de corte (IPC) são considerados três parâmetros: a variável de saída do método fuzzy, o número de consumidores VIPs e a geração de energia no alimentador, utilizando a equação (2).

$I P C=\left\{\begin{array}{l}\text { se } P_{\text {inst ALIM }}<0, I P C=S F+V A L O R_{M A X} V I P S+1 \\ \text { se } P_{\text {inst ALIM }} \geq 0, I P C=S F+V I P S\end{array}\right.$

Onde:

IPC é indicador de prioridade de corte.

$\mathrm{P}_{\text {instALIM }}$ é o valor da carga ativa instantânea do alimentador, em MW.

SF é o valor numérico da variável de saída do método fuzzy.

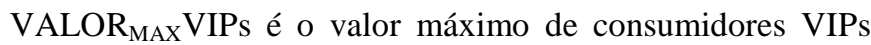
conectados entre todos os alimentadores.

VIPs é a quantidade de hospitais, consumidores residenciais com equipamento sobrevida e outras concessionárias ligadas ao alimentador.

A aplicação da equação (2) é baseada na heurística de que alimentadores que estão contribuindo com geração de energia ao sistema ficarão ao final da fila de classificação, após todos os outros alimentadores, possuindo carga prioritária (VIPs) ou não. Os alimentadores que não possuem cargas prioritárias ficarão no início da fila de classificação, pois são classificadas apenas pelo método fuzzy e tem adicionado 0 VIPs ao seu resultado.

\subsection{Seleção dos alimentadores para o corte}

Para a seleção dos alimentadores de acordo com os critérios estabelecidos pelo FPO, os alimentadores são agrupados por SE de acordo com o IPC. O valor dos cortes por barra é obtido através do método do item 2.1 e ordenado de maneira decrescente. As potências ativas instantâneas dos alimentadores são agrupadas até que se satisfaçam seus valores.

Como os valores do item 2.1 tem origem nos dados das barras e o corte é efetivado nos alimentadores, à medida que uma SE tem seu corte satisfeito, pode ser gerado um excedente de corte. Utilizando como base o trabalho de Sanaye-Pasand and Davarpanah (2005), afim de evitar um 
corte de carga desnecessário, quando o montante de carga solicitado pelo ONS é atingido, mais nenhuma SE tem sua carga cortada, mesmo que existam valores a cortar.

A Figura 3 apresenta um fluxograma do método de seleção dos alimentadores.

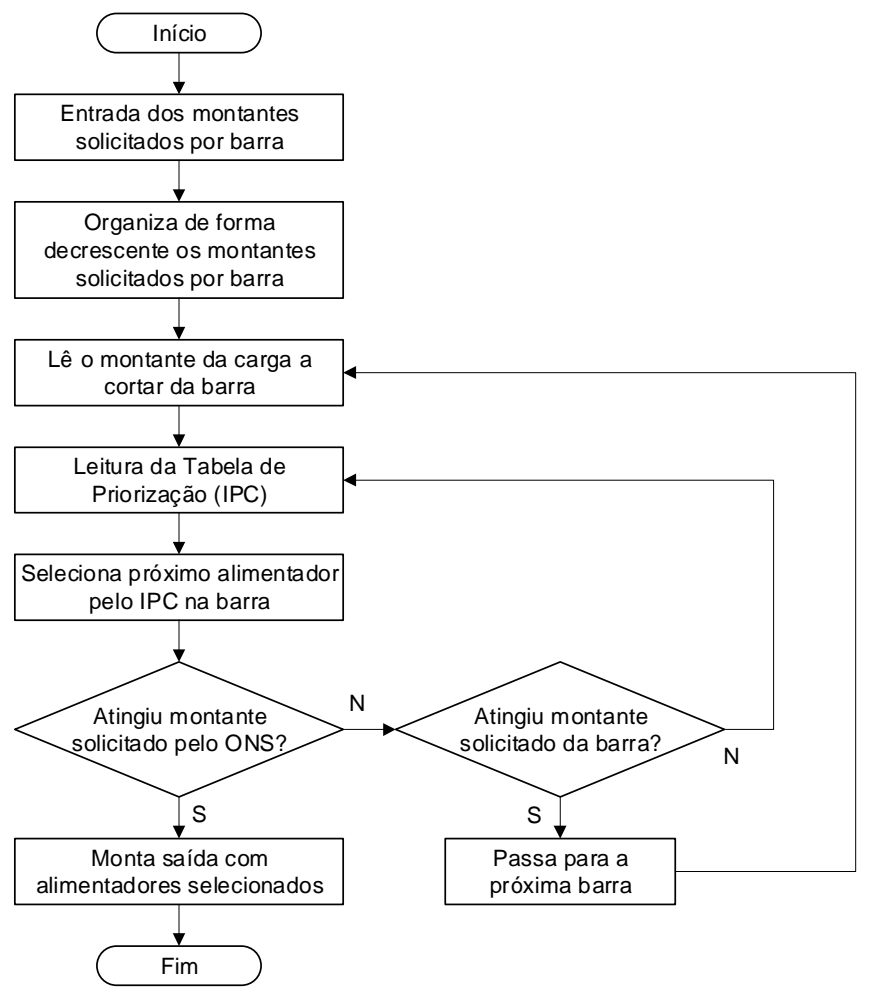

Fig. 3 Seleção dos alimentadores

\section{RESULTADOS}

Os resultados apresentados se mostraram satisfatórios. Utilizando os valores de corte de carga da Copel para o PCMC, segundo o Anexo 1 da IO-GC.BR.01, foram obtidos os valores da Tabela 1.

\section{Tabela 1. Resultado do corte de carga}

\begin{tabular}{|c|c|c|c|}
\hline $\begin{array}{c}\text { Solicitado } \\
\text { (MW) }\end{array}$ & $\begin{array}{c}\text { Corte } \\
\text { (MW) }\end{array}$ & SEs & Alimentadores \\
\hline 95 & 96,31 & 6 & 24 \\
\hline 114 & 118,25 & 8 & 28 \\
\hline 133 & 137,21 & 8 & 31 \\
\hline 152 & 155,93 & 10 & 36 \\
\hline 171 & 171,19 & 12 & 42 \\
\hline 190 & 190,26 & 13 & 55 \\
\hline 210 & 214,52 & 14 & 56 \\
\hline 229 & 230,88 & 15 & 56 \\
\hline 248 & 248,93 & 17 & 66 \\
\hline 267 & 274,30 & 18 & 73 \\
\hline 286 & 287,03 & 20 & 77 \\
\hline 305 & 316,03 & 21 & 85 \\
\hline 324 & 325,41 & 22 & 96 \\
\hline 343 & 345,56 & 23 & 104 \\
\hline 362 & 365,73 & 22 & 100 \\
\hline 381 & 390,11 & 23 & 112 \\
\hline
\end{tabular}

Utilizando o exemplo de $95 \mathrm{MW}$ de corte solicitado pelo ONS, obtêm-se os valores da Tabela 2 .

Tabela 2. Exemplo de corte de 95 MW

\begin{tabular}{|l|c|c|}
\hline Subestação & $\begin{array}{c}\text { Solicitado } \\
\text { FPO (MW) }\end{array}$ & $\begin{array}{c}\text { Efetivado } \\
\text { (MW) }\end{array}$ \\
\hline Medianeira & 25,41 & 33,21 \\
\hline Loanda & 18,51 & 20,94 \\
\hline Santa Helena & 14,40 & 17,39 \\
\hline Irati & 14,13 & 14,25 \\
\hline Imbituva & 5,83 & 6,30 \\
\hline Rio Azul & 4,73 & 4,23 \\
\hline Tafisa & 4,15 & - \\
\hline Ubiratã & 3,89 & - \\
\hline Mamborê & 2,58 & - \\
\hline Alto Paraná & 1,19 & - \\
\hline Cianorte & 0,18 & - \\
\hline Total & 95 & 96,32 \\
\hline
\end{tabular}

Como foi explicado no item 2.3 o excedente de carga cortado pelos alimentadores não foi propagado, interrompendo o corte após atingir o montante total solicitado. A Figura 4 apresenta um gráfico com o excedente percentual dos montantes de corte da Tabela 1 comparado com o método da divisão proporcional pela região geoelétrica.

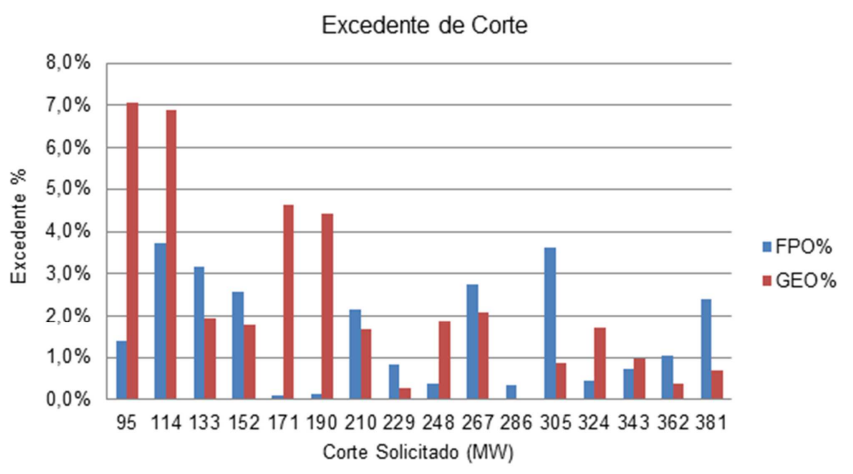

Fig. 4 Excedente de corte em função do corte solicitado

Em relação à utilização da lógica fuzzy para classificação dos alimentadores, esta também apresentou resultados satisfatórios, pois em conjunto com a equação (2), não houve nenhum alimentador com o mesmo IPC em uma mesma subestação, eliminando a necessidade de critério de desempate para seleção de alimentadores durante o processo de corte.

\section{CONCLUSÕES}

Para manter o balanço entre a demanda e a oferta de energia e evitar o colapso do sistema na ocorrência de imprevistos na operação em tempo real, os órgãos reguladores normatizam processos de controle envolvendo corte de carga, entre eles o PCMC, em que os agentes de distribuição devem efetivar o corte de carga em até cinco minutos após a solicitação.

$\mathrm{O}$ método proposto utilizando FPO + FUZZY obteve seus resultados alcançados, sendo que todos os cortes de carga foram atingidos, eliminando cortes desnecessários em subestações. 
A utilização de FPO para o corte de carga no PCMC apresenta um avanço nesta metodologia, que atualmente está baseada em uma divisão proporcional pela carga da região. A introdução de variáveis georreferenciadas, para evitar de maneira automática o corte em regiões centrais, e indicadores de continuidade, como CHI e CI, também apresenta avanço para a classificação de alimentadores, atualmente baseado em número de consumidores, pois amenizam os impactos do corte de carga e aumentam a isonomia entre os consumidores. Importante salientar a utilização de dados de potência instantânea dos alimentadores, fundamentais para minimizar o corte de carga.

Não foi encontrada na literatura uma metodologia que abordasse os aspectos dinâmicos e espaciais apresentados pelo método proposto, mas os trabalhos estudados foram importantes para compor o modelo desenvolvido, contribuindo com premissas de corte de carga, composição de classes de consumo e organização dos conjuntos fuzzy.

Este trabalho pode ser aprimorado com um estudo sobre os parâmetros do FPO e que utilize dados instantâneos de potência ativa e reativa nas barras das subestações para compatibilizar os montantes solicitados por este método com os valores disponíveis pelos alimentadores. Outra proposta é a utilização da Base de Dados Geográfica da Distribuidora (BDGD) para aquisição de maneira automática dos dados de entrada do método.

\section{AGRADECIMENTOS}

Este trabalho foi integralmente apoiado pela Companhia Paranaense de Energia (COPEL) dentro do Programa de Pesquisa e Desenvolvimento da Agência Nacional de Energia Elétrica (ANEEL) - projeto PD 2866-0404/2014. Os autores gentilmente agradecem à COPEL, ANEEL e ao Instituto de Tecnologia para o Desenvolvimento - Lactec, onde o projeto foi desenvolvido.

\section{REFERÊNCIAS}

ANEEL (2015) 'Manual de Controle Patrimonial do Setor Elétrico'. Available at: http://www.aneel.gov.br.

Bruch, M., Kuhn, M. and Schmid, G. (2011) 'Power Blackout Risks', Cro Forum, (November), p. 32.

Calderaro, V. et al. (2011) 'Fuzzy load-shedding strategy in distribution systems', International Conference on Intelligent Systems Design and Applications, ISDA, (5), pp. 319-324. doi: 10.1109/ISDA.2011.6121675.

EPE (2018) 'Balanço Energético Nacional 2018'. Available at: http://www.epe.gov.br.

Ganeshprasad, G. G. et al. (2013) 'Time priority based optimal load shedding using genetic algorithm', in Fifth International Conference on Advances in Recent Technologies in Communication and Computing (ARTCom 2013). Institution of Engineering and Technology, pp. 301-308. doi: 10.1049/cp.2013.2187.

Mikilita, M. A. S. (2005) Um modelo de fluxo de potência ótimo para minimização do corte de carga. UFPR.

ONS (2016) 'MPO Módulo 10 - Submódulo 10.10 Gerenciamento da Carga', pp. 1-10. Available at: http://www.ons.org.br/.
ONS (2018) 'MPO Módulo 10 - Submódulo 10.21 Gerenciamento da Carga para Controle de Frequência no Sistema', pp. 1-6. Available at: http://www.ons.org.br/.

Reddy, G. H. et al. (2017) 'Prioritization of load points in distribution system considering multiple load types using fuzzy theory', IEEE International Conference on Fuzzy Systems, pp. 1-6. doi: 10.1109/FUZZIEEE.2017.8015535.

Sanaye-Pasand, M. and Davarpanah, M. (2005) 'A new adaptive multidimensioanal load shedding scheme using genetic algorithm', in Canadian Conference on Electrical and Computer Engineering, 2005. IEEE, pp. 1974-1977. doi: 10.1109/CCECE.2005.1557370.

Silva, A. C. da, Marques, R. and Jardim, M. da C. (2016) 'Esquema de Corte para Alívio de Carga', in XXII Seminário Nacional de Distribuição de Energia Elétrica. Curitiba.

Veloza, O. P. and Santamaria, F. (2016) 'Analysis of major blackouts from 2003 to 2015: Classification of incidents and review of main causes', Electricity Journal. Elsevier Inc., 29(7), pp. 42-49. doi: 10.1016/j.tej.2016.08.006.

Vieira, S. K. B. et al. (2016) 'Plano de Corte Manual de Carga', in XXII Seminário Nacional de Distribuição de Energia Elétrica. Curitiba, pp. 1-12. 